\title{
Painlevé-Kuratowski convergences of the approximate solution sets for vector quasiequilibrium problems
}

\author{
NGUYEN VAN Hung ${ }^{1}$, Dinh Huy HoAng ${ }^{2}$ and Vo Minh TAM ${ }^{1}$
}

\begin{abstract}
.
In this paper, we study vector quasiequilibrium problems. After that, the Painlevé-Kuratowski upper convergence, lower convergence and convergence of the approximate solution sets for these problems are investigated by using a sequence of mappings $\Gamma_{C}$-converging. As applications, we also consider the Painlevé-Kuratowski upper convergence of the approximate solution sets in the special cases of variational inequality problems of the Minty type and Stampacchia type. The results presented in this paper extend and improve some main results in the literature.
\end{abstract}

Acknowledgements. This research was supported by Ministry of Education and Training of Vietnam under grant number B2017.SPD01. The authors are grateful to Prof. Phan Quoc Khanh, Prof. Lam Quoc Anh and two anonymous referees for their valuable remarks and suggestions, which helped to improve the paper.

\section{REFERENCES}

[1] Anh, L. Q. and Hung, N. V., Stability of solution mappings for parametric bilevel vector equilibrium problems, Comp. Appl. Math., (2016), online first

[2] Anh, L. Q. and Hung, N. V., Gap functions and Hausdorff continuity of solution mappings to parametric strong vector quasiequilibrium problems, J. Ind. Manag. Optim., 14 (2017), 65-79

[3] Fang, Z. M. and Li, S. J., Painlevé-Kuratowski convergences of the solution sets to perturbed generalized systems, Acta Math Appl. Sin-E., 2 (2012), 361-370

[4] Fang, Z. M., Li, S. J. and Teo, K. L., Painlev é-Kuratowski convergences for the solution sets of set-valued weak vector variational inequalities, J. Inequal. Appl., ID 43519 (2008), 1-14

[5] Lalitha, C. S. and Chatterjee, P., Stability and scalarization in vector optimization using improvement sets, J. Optim. Theory Appl., 166 (2015), 825-843

[6] Li, X. B. and Li, S. J., Continuity of approximate solution mapping for parametric equilibrium problems, J. Glob. Optim., 51 (2011), 541-548

[7] Li, X. B., Lin, Z. and Wang, Q. L., Stability of approximate solution mappings for generalized Ky Fan inequality, TOP, 24 (2015), 196-205

[8] Li, X. B., Long, X. J. and Zeng, J., Holder continuity of the solution set of the Ky Fan inequality, J. Optim. Theory Appl., 158 (2013), 397-409

[9] Long, X. J., Huang, N. J. and Teo, K. L., Existence and stability of solutions for generalized strong vector quasiequilibrium problem, Math. Comput. Model., 47 (2008), 445-451

[10] Luc, D. T, Lucchetti, R. E. and Maliverti, C., Convergence of the efficient sets. Set convergence in nonlinear analysis and optimization, Set-Valued Anal., 2 (1994), 207-218

[11] Lucchetti, R. E. and Miglierina, E., Stability for convex vector optimization problems, Optimization, 53 (2004), $517-528$

[12] Maso, G. D., An Introduction to $\Gamma$-Convergence, Progress in Nonlinear Differential Equations and their Applications, Vol. 8, Birkhaẽ user Boston Inc., Boston, MA (1993)

Received: 05.03.2017; In revised form: 05.12.2017; Accepted: 12.12.2017

2010 Mathematics Subject Classification. 90C31, 49K40, 91B50, $49 \mathrm{~J} 45$.

Key words and phrases. Quasiequilibrium problems, quasivariational inequality problems; approximate solution,

Painlevé-Kuratowski convergence; $\Gamma_{C}$-convergence, continuous convergence.

Corresponding author: Nguyen Van Hung; ngvhungdhdt@yahoo.com 
[13] Oppezzi, P. and Rossi, A. M., A convergence for vector-valued functions, Optimization, 57 (2008), 435-448

[14] Oppezzi, P. and Rossi, A. M., A convergence for infinite dimensional vector valued functions, J. Glob. Optim., 42 (2008), 577-586

[15] Oppezzi, P. and Rossi, A. M., Existence and convergence of Pareto minima, J. Optim. Theory Appl., 128 (2006), 653-664

[16] Peng, Z. Y. and Yang, X. M., Painleve-Kuratowski convergences of the solution set for perturbed vector equilibrium problems without monotonicity, Acta Math. Appl. Sin. Engl. Ser., 30 (2014), 845-858

[17] Peng, Z. Y., Yang, X. M. and Peng, J. W., On the lower semicontinuity of the solution mappings to parametric weak generalized Ky Fan inequality, J. Optim. Theory Appl., 152 (2012), 256-264

[18] Rockafellar, R. T. and Wets, R. J., Variational analysis, Springer, Berlin (1998)

[19] Song, Q. Q. and Wang, L. S., On the stability of the solution for multiobjective generalized games with the payoffs perturbed, Nonlinear Anal. TMA., 73 (2010), 2680-2685

[20] Song, Q. Q., Yang, G. H. and Zhong, C. Y., On the stability of finding approximate fixed points by simplicial methods, J. Inequal. Appl., (2016), 2016:220

[21] Zhao, Y., Peng, Z. Y. and Yang, X. M., Painlevé-Kuratowski convergences of the solution sets for perturbed generalized systems, J. Non. Convex Anal., 15 (2014), 1249-1259

\author{
${ }^{1}$ Department of Mathematics \\ DONG THAP UNIVERSITY \\ DONG THAP, VIETNAM \\ E-mail address: ngvhungdhdt @yahoo.com \\ E-mail address: vmtamedthu.edu.vn \\ 2 Department of Mathematics \\ VINH UNIVERSITY \\ NGHE AN, VIETNAM \\ E-mail address: dhhoangdhv@gmail .com
}

